\title{
Monocyte Activation Test (MAT) as a possibility of replacement for the rabbit pyrogen test in hyperimmune sera
}

\author{
Carla Lilian de Agostini Utescher,, Klariane Laís Buosi², Viviane Fungaro Botosso', \\ Wagner Quintilio ${ }^{3}$
}

\begin{abstract}
${ }^{1}$ Virology Laboratory, Instituto Butantan, São Paulo, SP, Brazil, ${ }^{2}$ Application Specialist, Merck SA, São Paulo, SP, Brazil, ${ }^{3}$ Laboratory of Biopharmaceuticals in animal cells, Instituto Butantan, São Paulo, SP, Brazil
\end{abstract}

\begin{abstract}
The use of a commercial kit for the monocyte-activation test (MAT) was evaluated for assessing pyrogenic contamination of hyperimmune sera. Three batches of sera, two pyrogen free and one pyrogenic, were tested. Endotoxin spike recover indicated that sample dilutions from 1/2 to $1 / 10$ are suitable. Kit transport and storage conditions were also evaluated, proving that an adequate cold chain must be assured to achieve good results. Furthermore, the commercial MAT kit seemed suitable to replace the rabbit pyrogen test (RPT) for pyrogen testing of hyperimmune sera, although further tests are needed to a full validation.
\end{abstract}

Keywords: Monocytes Activation Test. Pyrogen. Hyperimmune serum. Pyrodetect kit. Cryoblood.

\section{INTRODUCTION}

Pyrogens can be roughly defined as substances, derived from microorganisms, that induce fever in animals (Hartung, 2015). Lipopolysaccharide (LPS), lipoteichoic acid LTA (Morath, Geyer, Hartung, 2001), exotoxins (Blackman, Woodland, 1995), muramyldipeptide (Nakatani et al., 2002) and peptidoglycan (Schleifer, 1975) are examples of pyrogens whose ubiquity is a threat to human health due to their stability (Schindler et al., 2006).

Whenever a pyrogenic substance enters in the bloodstream, it stimulates innate immunity leading to the secretion of fever-inducing cytokines . The most important are Interleukin-1 $\beta$ (IL-1 $\beta$ ), Interleukin-6 (IL-6), Tumor Necrosis Factor-a (TNF-a) and Interferon (IFN-g), which act in the thermoregulatory center of the organism, mediating a rise in body temperature (Schindler et al., 2006).

All parenteral products for human use must be pyrogen free and Quality Control assessing pyrogenic contamination is mandatory (Brazilian Phamacopoeia, 2010). In the beginning of the $20^{\text {th }}$ century, a pyrogen test in rabbits (Rabbitt Pyrogen Test - RPT) was developed

\footnotetext{
*Correspondence: C. L. A. Utescher. Laboratório de Virologia, Instituto Butantan. Av Dr Vital Brazil, 1500 05503-000 São Paulo, SP - Brasil. Tel. +55 11 2627-9869. E-mail: carla.utescher@butantan.gov.br
}

and since then it has been used to ensure the safety of biologicals (Hasiwa et al., 2013). However RPT is not suitable for certain pharmaceutical products - such as radio or chemotherapeutics, analgesics, steroids, cytokines, dopamine and immunosuppressive agents (Greenhough, 2016) - and, as are all animal tests, it is expensive, prone to errors and ethically questionable. The bacterial endotoxin test (BET) - also called Limulus Amebocyte Lysate test (LAL) - was developed later based on the observation that the hemolymph of horseshoe crabs clots in the presence of endotoxins (Hasiwa et al., 2013). Considering the variety of existing pyrogens, BET should only be used to replace RPT when the potential contaminants are endotoxins. Even though, several plasma fractions or plasma derived medicinal products may inherently contain constituents which interfere with different LAL testing methods (EMEA, 2007; Potu, Burra, Patil, 2011). It has also been demonstrated that in certain biopharmaceutical product formulations containing citrate, phosphate and polysorbate the endotoxins can be masked and not detected by BET (Bolden et al., 2014).

Due to the importance of reducing animal testing for the quality control of biologicals in light of the 3 R's principle, alternatives to RPT were developed taking into account the fever induction mechanism of pyrogeninduced cytokines. The monocyte activation test (MAT), for example, is based on the detection of IL- 6 or IL-1b 
induced in human blood monocytes or monocytic cell lines stimulated in vitro by pyrogens (detailed by Peterbauer $e t$ $a l ., 1999 ; 2000)$. The test was validated in an international collaborative study (Schindler et al., 2006) and in 2010 the MAT was incorporated in the European Pharmacopoeia (European Pharmacopoeia, 2010).

The most important drawback for the MAT is the need to use human blood with viable monocytes whose half-life is less than 2 hours in vitro. In addition, according to Brazilian rules, donated blood is the property of the federal government intended only for transfusions or for the purification of blood fractions (Brasil, 2001), besides biosafety concerns. In order to overcome the limitations related to the use of fresh blood, a cryopreservation method was developed, where the blood could be used immediately after thawing (Schindler et al., 2006). Commercially, cryopreserved blood is readily available, simplifying the MAT standardization. The commercially available MAT kit uses cryopreserved human blood as a source of monocytes and the response to pyrogenic substances is determined by measurement of the induced Interleukin - $1 \beta$ (IL-1 $\beta$ ) by ELISA.

Hyperimmune sera are an important class of biological products constituted of purified specific $F(a b ') 2$ fragments produced in immunized horse. Antivenom sera are used for the treatment snakebite envenomation anticrotalus, for instance - or in the treatment of patients with diseases caused by microorganisms (bacteria or virus), using antisera such as antitetanus, antidiphtheria or antirabies. Contamination with microbial molecules and protein aggregates, due to high protein concentration, during the production process may be the reason for the pyrogenicity that cannot be detected by BET (Schindler et al., 2006).

The US Interagency Coordinating Committee for the Validation of Alternative Test Methods (ICCVAM) declared that "MAT is suitable after a product-specific validation as a replacement for the Rabbit Pyrogen Test (RPT)" (ICCVAM, 2008). But it must be validated for each product matrix (Bolden et al., 2014), specially to prove that inherent components will not interfere, resulting in errors.

The aim of this work was to evaluate the use of this commercial MAT kit as part of an effort to replace RPT of hyperimmune sera produced by Instituto Butantan, taking into account standardization and ease of use in a routine testing environment.

The study was based on LPS recovery and the capacity to detect pyrogenicity in a pyrogen positive sample (as previously determined by RPT). Blood cryopreservation and transport conditions were also tested in order to assess the kit's viability considering a routine use in quality control.

\section{MATERIAL AND METHODS}

\section{Study design}

This study consists of the evaluation of test suitability to detect pyrogens in hyperimmune sera. At least three individual tests were performed with known samples to evaluate equivalence and accuracy (spiking the sample with standard endotoxin). The relationship between kit transport conditions and test validity was also evaluated considering the kit temperature sensitivity.

\section{Samples}

Three batches of hyperimmune sera were used in this study: one anticrotalus serum (ACS) and one antidiphtheria serum (ADS), both non-pyrogenic; and one antitetanus serum (ATS), positive for pyrogens as determined by RPT. All samples were kindly provided by the Division of Scientific Development and Production of Instituto Butantan.

\section{Standards}

LPS (E. coli O113:H10:K-endotoxin) standard BRP batch 5 supplied by EDQM (European Directorate for the Quality of Medicines and HealthCare European Pharmacopoeia) and distributed by Merck was reconstituted with non-pyrogenic water and stored at $-80{ }^{\circ} \mathrm{C}$ until use. Standard interleukin- $1 \beta$ used as positive control in ELISA was supplied by Merck. Unless otherwise stated, all reagents were used according to supplier instructions.

\section{Rabbit Pyrogen Test - RPT}

No animals were used in this study; the samples were tested in rabbits by the internal quality control of Instituto Butantan, in accordance with the Brazilian Pharmacopoeia recommendations for the routine lot release (Brazilian Pharmacopoeia, 2010).

\section{PyroDetect System}

The MAT system supplied by Merck consists of the PyroDetect Kit (ELISA kit to detect human IL-1 $\beta$ ), Cryoblood (cryopreserved human blood), standard IL-1 $\beta$ (ELISA positive control) and Standard LPS. The detection 
system was used following the manufacturer instructions. The assay was performed in two steps. Briefly, incubation with blood (fresh or cryopreserved) and ELISA to detect induced IL-1 $\beta$. Samples and standard endotoxin were incubated with cryopreserved blood $\left(16-20 \mathrm{~h}, 37^{\circ} \mathrm{C}, 5 \%\right.$ $\mathrm{CO}_{2}$ ) under sterile conditions. The mixtures were then transferred to ELISA microplates coated with monoclonal antibody anti-IL-1 $\beta(2 \mathrm{~h}$, room temperature - RT). After washing, horseradish peroxidase labeled anti-human IL-1 $\beta$ was added ( $1 \mathrm{~h}, \mathrm{RT})$. The microplate was washed and enzyme substrate was added (20 min, RT). The reaction was stopped with $\mathrm{H}_{2} \mathrm{SO}_{4}$ and the absorbance was recorded at $450 \mathrm{~nm}$ (reference at $630 \mathrm{~nm}$ ). Regression and linearity of the standard curves were evaluated by using Combistats 4.0.

Limit of detection (LOD - average absorbance of four independent negative control samples increased by 3 -fold the standard deviation and interpolated in the standard curve) was calculated by using GraphPad Prism ${ }^{\circledR}$ 5.01 .

\section{Endotoxin recovery testing}

In order to evaluate endotoxin recovery rates, the samples of antisera were diluted 1/2,1/4, 1/8 and 1/10 in RPMI (Roswell Park Memorial Institute) medium (part of the PyroDetect kit) and submitted to the MAT either diluted uncontaminated or contaminated with $0.5 \mathrm{EU} /$ $\mathrm{mL}$ of standard endotoxin. Through the standard curve, endotoxin recovery was calculated by using GraphPad Prism 5.01 software, considering the Asymmetric sigmoidal model. The endotoxin recovery test was performed 3 times for dilutions $1 / 2,1 / 4$ and $1 / 8$, and 4 times for dilution $1 / 10$. The ANOVA test was used to compare experimental groups. Relative standard deviation (RSD\% - $100 \times$ ratio standard deviation/mean) was calculated for all data groups.

\section{Cryoblood transport}

Cryoblood is a cryopreserved standardized human blood preparation (certified pool of 8-10 donors). It must be transported and stored at temperatures lower than $80^{\circ} \mathrm{C}$ as indicated in the product notice.

\section{Maximum Valid Dilution (MVD) calculation}

In order to define the maximum dilution at which pyrogens can still be detected, MVD was calculated in accordance with the formula defined in the European Pharmacopoeia (European Pharmacopoeia, 2010):

\section{$\mathrm{MVD}=\mathrm{CLC} \times \mathrm{C} / \mathrm{LOD}$}

where: $\mathrm{CLC}=$ contaminant limit concentration; $\mathrm{C}=$ concentration of test solution; $\mathrm{LOD}=$ Limit of detection of the test: $0.25 \mathrm{EU} / \mathrm{mL}$; Endotoxin limit concentration for parenteral drugs: $5 \mathrm{EU} / \mathrm{kg} / \mathrm{h}$ (European Pharmacopoeia, 2010); - Maximum dose for each serum is specified in the respective product notice or in the user's recommendations published by the National Authority, namely: ACS - $200 \mathrm{~mL} / 70 \mathrm{~kg} / 24 \mathrm{~h}$; ADS - $120 \mathrm{~mL} / 70 \mathrm{~kg} / 24 \mathrm{~h}$; ATS - $20 \mathrm{~mL} / 70 \mathrm{~kg} / 24 \mathrm{~h}$.

\section{RESULTS}

\section{Endotoxin standard curve}

Endotoxin standard curves were prepared for five different cryoblood lots. The difference between these lots regarded the transport conditions. All kit lots were transported in dry ice, though through different routes. Some were transported under rigid temperature control while others were not monitored and transported under less rigid temperature conditions. Two curves were prepared for the transport at a rigidly controlled and monitored temperature (more than $90 \%$ of the time the temperature was lower than $70{ }^{\circ} \mathrm{C}$ ), and three for the transport at no rigid temperature conditions (Figure 1), herein called unmonitored. When the Cryoblood is transported at unmonitored, hence unknown, conditions (although after its arrival in the laboratory it was stored at temperatures lower than $-80^{\circ} \mathrm{C}$ ), the results were lower than expected (Figure 1). Calculated LOD ranged from $0.010 \mathrm{EU} / \mathrm{mL}$ (curve 5) to $0.423 \mathrm{EU} / \mathrm{mL}$ (curve 3) (Table I). However, curves 2 and 3 were considered invalid due to their highest LOD (above $0.250 \mathrm{EU} / \mathrm{mL}$ ), hence they were not considered in further analyses.

\section{Endotoxin recovery}

The diphtheria antiserum, the tetanus antiserum and the crotalus antiserum were tested to assess the endotoxin recovery rate. In order to establish the better sample dilution to test performance, meaning the one or those whose recovery is in the range $50-200 \%$ and the Relative standard variation (precision) is as low as possible, the endotoxin recovery rate was plotted against sample dilutions (Figure 2). Using ANOVA, no significant differences were observed among the dilutions $(\mathrm{p}>0.05)$. However, when the sample was tested pure undiluted, recovery was lower than with the diluted samples, outside of the limit range (50-200\%). 
TABLE I - Regression and non-linearity p values calculated for endotoxin standard curves as validity criteria. Cut-off and Limit of detection (LOD) calculated for each test

\begin{tabular}{cccccc}
\hline Curve & $\boldsymbol{p}$ for regression & $\boldsymbol{p}$ for non-linearity & Cut-off & LOD $(\mathbf{E U} / \mathbf{m L})$ & Validity \\
\hline 1 & 0.001 & 0.955 & 0.174 & 0.179 & Valid \\
2 & 0.004 & 0.398 & 0.035 & 0.269 & Invalid \\
3 & 0.018 & 0.980 & 0.087 & 0.423 & Invalid \\
4 & 0.000 & 0.969 & 0.511 & 0.107 & Valid \\
5 & 0.000 & 0.843 & 0.621 & 0.010 & Valid \\
\hline
\end{tabular}

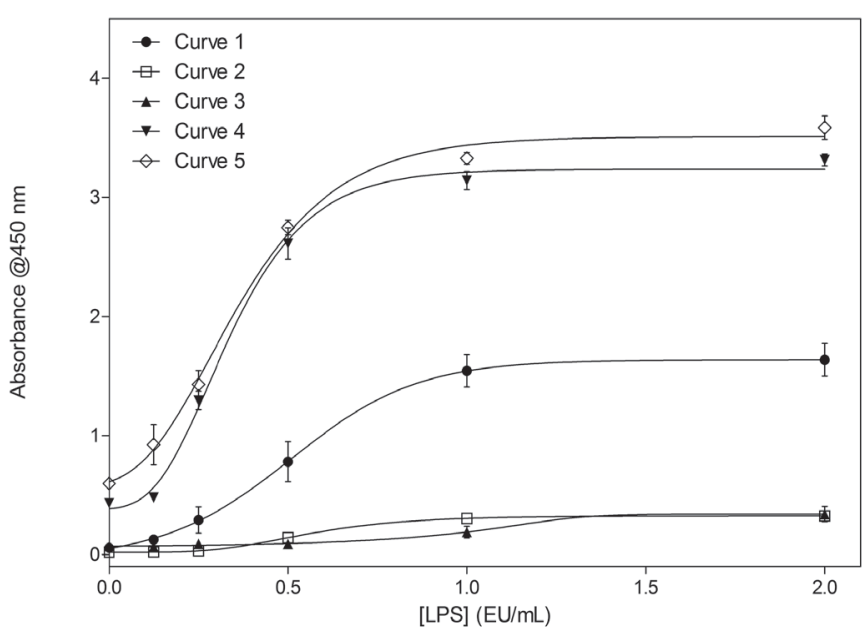

FIGURE 1 - Endotoxin standard curves obtained with cryoblood transported under different conditions. Unmonitored (Curves 1, 2 and 3 ) and monitored at a stable temperature (curves 4 and 5).

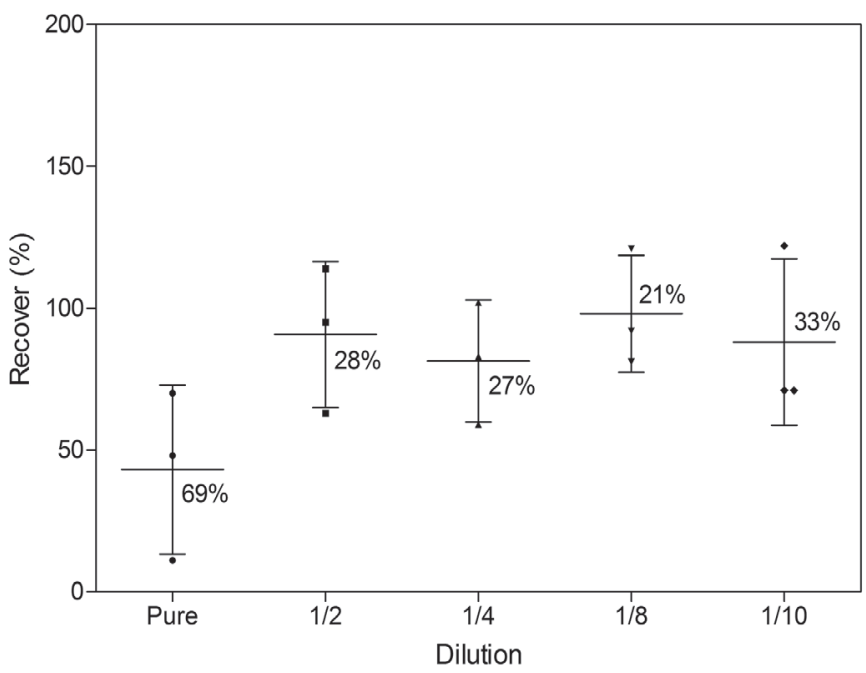

FIGURE 2 - Endotoxin recovery rate (\%) of the undiluted antiserum, the diluted serum and the antiserum spiked with standard endotoxin $(0.5 \mathrm{EU} / \mathrm{mL})$. The recovery rate was calculated by interpolation from the standard curve subtracting the endotoxin equivalent of the non-spiked sample. The numbers indicate the relative standard deviation for each dilution. There were no statistically significant differences among the groups.

\section{MVD calculation for tested antisera}

MVD depends on the type of antiserum, because concentrations and uses vary according to the treatment conditions. Calculated values of MVD were: ACS: 168; ADS: 280 and ATS: 1680 all of them above the dilutions used in this study.

\section{Monocyte Activation Test (MAT)}

Using the valid standard endotoxin curves (Figure 1, Table I), the endotoxin recovery rate was calculated for ADS (diluted 1/8), ATS and ACS (diluted 1/10); all results were within the required range of 50-200\%. As expected, the non-spiked ATS resulted positive for pyrogen, i.e., above the limit of detection of the standard curve (Table II).

\section{DISCUSSION}

The suitability of a MAT commercial kit for pyrogen testing in hyperimmune serum samples was evaluated. Three different samples, positive and negative for pyrogens, were tested using the kit; in our experimental conditions, the presence of pyrogens was easily and accurately verified. The lowest RSD values in the endotoxin recovery rates were observed when the samples were diluted 1/2 until 1/10 (Figure 2) and sample dilution did not interfere with the endotoxin detection. These dilutions were lower than the maximum dilution value (MDV) for each sample, avoiding false-negatives due to high dilution. The commercial MAT kit used following the manufacturer's recommended conditions successfully detected pyrogenicity (in endotoxin equivalent units $\mathrm{EEU} / \mathrm{mL}$ ) in the pyrogenic sample.

The MAT commercial kit is easy to use but as a bacterial endotoxin is used as for the standard curve, the results depend on its suitable handling. Well-trained technicians would not have any difficulty performing the test adequately.

Animal testing for quality control of biologicals is 
TABLE II - Results for MAT performed with Cryoblood transported and stored under monitored and controlled conditions. The results for equivalent endotoxin and recovery rates are averages of quadruplicates. Samples were diluted 1/10 and the represented values are average of quadruplicates. ATS: tetanus antiserum; ADS: diphtheria antiserum; ACS: crotalus antiserum

\begin{tabular}{ccccc}
\hline \multirow{2}{*}{ Sample } & $\begin{array}{c}\text { Endotoxin standard } \\
\text { curve }\end{array}$ & \multicolumn{2}{c}{ EEU/mL } & \multirow{2}{*}{ Recover (\%) } \\
\cline { 3 - 4 } & 1 & Non-spiked & spiked & 92 \\
ADS & 4 & 0 & 0.4610 & 122 \\
ATS & 5 & 0.1380 & 0.7500 & 71 \\
ACS & & 0 & 0.3532 & 71 \\
ATS & & 0.2627 & 0.6189 & 92 \\
\hline
\end{tabular}

living on borrowed time, depending only on the scientific community efforts to validate and implement alternatives. RPT is an important and mandatory safety test for some biologicals (Brazilian Pharmacopoeia, 2010), notably hyperimmune sera, but besides the ethical issues, rabbits are expensive animals to obtain and keep and for this test reuse is not recommended (Brazilian Phamacopoeia, 2010). Established alternatives, such as BET, are mostly indicated for the control of water for injection, but is not suitable to warrant serum safety. In Brazil, unfortunately, an alternative to RPT is still not available. In order to reduce the total number of animals for batch control, an interesting approach might be the use of MAT for in-process control, leaving RPT just for the final product until the alternative test is fully validated and accepted by the national authority.

The purpose of this work was not to replace the method validation study, once only few parameters could be evaluated. Full validation should also include robustness and large sample number in order to check precision and accuracy (ICCVAM, 2008), in addition to spiking with other known pyrogens, such as lipotheicoic acid (Da Silva et al., 2016). However, this kind of study may become impracticable if kit transport and storage conditions are not properly monitored or controlled as demanded by the kit notice and as demonstrated in this study (Figure 1, Table I). The main advantage of using a commercial kit is the standardization of the reagents and, in this case, specifically cryopreserved blood. Since the use of fresh blood may be proove difficult for logistic or even legal issues (Brasil, 2001), commercial kits containing cryopreserved standardized blood are an interesting alternative, which could lead to the successful replacement of RPT. This available commercial blood consists of a standardized pool of 8-10 donors, certified, which means it might be an interesting advantage from thequality assurance point of view. Nevertheless, the drawback of this approach is the need for an appropriate cold chain maintenance to allow the use of cryopreserved blood, which could be an issue for developing countries.
This became clear during this study. Kit transportation and storage must be carefully managed respecting reagents temperature sensitivity in order to avoid kit instability, ultimately leading to test invalidity (Figure 1).

The test showed equivalence to RPT and detected pyrogens in the tested samples. Although a full validation study is still mandatory for the test to be implemented in a quality control routine, this study demonstrates that the MAT commercial kit is suitable for the detection of pyrogen in hyperimmune serum samples.

\section{ACKNOWLEDGMENTS}

The authors would like to acknowledge Dr Celso Pereira Caricati for the kind and helpful support and DDTP for the antisera supply. Financial support by Fundação Butantan and CNPq (grant 403299/2012-8).

\section{CONFLICT OF INTEREST}

KL Buosi is application specialist at Merck SA, supplier of the Pyrodetect kit.

\section{AUTHOR CONTRIBUTIONS}

CLAU collected and analyzed data and wrote the manuscript; KLB and VFB wrote the manuscript; WQ idealized the project, collected and analyzed data and wrote the manuscript.

\section{REFERENCES}

Blackman MA, Woodland DL. In vivo effects of superantigens. Life Sci. 1995;57(19):1717-35.

Bolden JS, Claerbout ME, Miner MK, Murphy MA, Smith KR, Warburton RE. Evidence against a bacterial endotoxin masking effect in biologic drug products by limulus amebocyte lysate detection. PDA J Pharm Sci Technol. 2014;68(5):472-7. 
Brasil. Lei n ${ }^{\circ}$ 10.205, de 21 de março de 2001. [citado em 01 Dec 2017]. Disponível em: http://www.planalto.gov.br/ccivil_03/ leis/leis_2001/110205.htm

Brazilian Pharmacopoeia. 5 ed. Brasília: Anvisa; 2010. Part. 2. da Silva CC, Presgrave OA, Hartung T, de Moraes AM, Delgado IF. Applicability of the Monocyte Activation Test (MAT) for hyperimmune sera in the routine of the quality control laboratory: Comparison with the Rabbit Pyrogen Test (RPT). Toxicol In Vitro. 2016;32:70-5.

European Medicines Agency (EMEA), Replacement of rabbit pyrogen testing by an alternative test for plasma derived medicinal products. 2007. [citado em 01 DEC 2017]. Disponível em: http://www.ema.europa.eu/docs/en_GB/document_library/ Scientific_guideline/2009/09/WC500003599.pdf.

European Pharmacopeia. 7. ed. Monocyte activation test. General Method. Strasbourg: Council of Europe; 2010. Chap. 2.6.30.

Greenhough J. Life Cycle of an analytical method: a case study on the monocyte activation test. 2016. [citado em 01 Dec 2017]. Available on: http://www.wickhamlabs.co.uk/wp-content/ uploads/2016/12/Wickham-Laboratories-Case-Study-on-theMonocyte-Activation-Test.pdf.

Hartung, T. The human blood pyrogen test - lessons learned in twenty years. ALTEX. 2015;32(2):79-100.

Hasiwa N, Daneshian M, Bruegger P, Fennrich S, Hochadel A, Hoffmann S, et al. Evidence for the detection of non-endotoxin pyrogens by the whole blood monocyte activation test. ALTEX. 2013;30(2):169-208.

Interagency Coordinating Committee on the Validation of Alternative Methods. ICCVAM. ICCVAM test method evaluation report: validation status of five in vitro test methods proposed for assesssing potential pyrogenicity of pharmaceuticals and other products. 2008. [citado em 01 DEC 2017]. Available from: https://ntp.niehs.nih.gov/iccvam/docs/ pyrogen/tmer/pyrotmer2008.pdf.
Morath S, Geyer A, Hartung T. Structure-function relationship of cytokine induction by lipoteicoic acid from Staphylococcus aureus. J Exp Med. 2001;193(3):393-397.

Nakatani T, Tsuchida K, Sugimura K, Yoshimura R, Takemoto Y. Response of peripheral blood mononuclear cells in hemodialyzed patients against endotoxin and muramyldipeptide. Int J Mol Med. 2002;10(4):469-72.

Peterbauer A, Eperon S, Jungi TW, Werner ER, WernerFelmayer G. Interferon-gamma-primed monocytoid cell lines: optimizing their use for in vitro detection of bacterial pyrogens. J Immunol Methods. 2000;233(1-2):67-76.

Peterbauer A, Werner ER, Werner-Felmayer G. Further development of a cell culture model for the detection of bacterial pyrogens. ALTEX. 1999;16(1):3-8.

Potu A, Burra S, Patil AK. Monocyte activation test: a new pharmacoepial quality control test for pyrogens: a review. J Adv Pharm Sci. 2011;1(1):122-131.

Schindler S, Spreitzer I, Löschner B, Hoffmann S, Hennes $\mathrm{K}$, Halder $\mathrm{M}$, et al. International validation of pyrogen tests based on cryopreserved human primary blood cells. J Immunol Methods. 2006;316(1-2):42-51.

Schleifer KH. Chemical structure of the peptidoglycan, its modifiability and relation to the biological activity. Z Immunitatsforsch Exp Klin Immunol. 1975;149(2-4):104-17.

Received for publication on $30^{\text {th }}$ August 2017 Accepted for publication on $23^{\text {th }}$ October 2017 